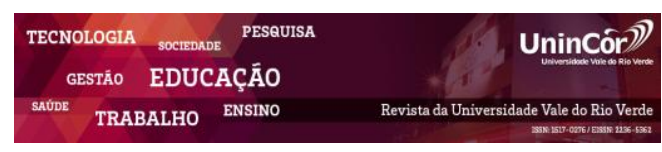

Revista da Universidade Vale do Rio Verde ISSN: 1517-0276 / EISSN: 2236-5362 v. $17 \mid$ n. 1 | Ano 2019

Raíssa Costa de Oliveira Universidade Federal do Ceará raissacostaufc@yahoo.com.br

Francisco Belmino Romero Universidade Federal do Ceará belmino@ufc.br

Carlucio Roberto Alves Universidade Estadual do Ceará alvescr@yahoo.com

Atanu Biswas

USDA Agricultural Research Service atanu.biswas@ars.usda.gov

Huai N.Cheng USDA Agricultural Research Service hn.cheng@ars.usda.gov

Roselayne Ferro Furtado Embrapa Agroindústria Tropical roselayne.furtado@embrapa.br

\section{FILMES DE POLIANILINA (PANI) \\ ELETROPOLIMERIZADOS SOBRE \\ SUPERFÍCIE DE ITO PARA USO COMO \\ INDICADOR COLORIMÉTRICO PARA DETECÇÃO DA AMÔNIA; \\ POLIMERIZAÇÃO DE FILMES DE PANI \\ COMO INDICADOR DA PRESENÇA DE AMÕNIA}

\begin{abstract}
RESUMO
Indicadores colorimétricos são sistemas que podem facilmente ser introduzidos a embalagens, a fim de informar ao consumidor a cerca da qualidade do produto. Amônia é gerada durante a deterioração do pescado e pode ser monitorada por meio de indicadores colorimétricos. Neste trabalho objetivou-se eletrossintetizar filmes de polianilina para a detecção da amônia por meio da mudança de cor. Filmes foram eletrodepositados por voltametria cíclica e cronoamperometria em meio ácido sobre superfície de ITO. O filme crescido por voltametria cíclica apresentou uma morfologia mais plana e um limite de detecção de $0,05 \mathrm{~mol} \mathrm{~L}^{-1}$. O filme crescido por cronoamperometria apresentou uma morfologia de rede interconectada, um limite de detecção de $0,015 \mathrm{~mol} \mathrm{~L}^{-1}$ e uma mudança de cor verde para azul mais perceptível visualmente. Assim, recomenda-se produzir o filme por cronoamperometria para a produção de um indicador colorimétrico para detecção da amônia em função do limite de detecção e da melhor visualização da mudança de coloração.
\end{abstract}

Palavras-chave: Anilina. Polimerização. Indicador. Mudança de cor. Amônia.

\begin{abstract}
Colorimetric indicators are systems easily introduced into packaging in order to inform the consumer about the quality of the product. Ammonia is generated during the fish deterioration and it can be monitored by using colorimetric indicators. In this work the objective was the electrosynthesis polyaniline films for the detection of ammonia by color change. Films were electrodeposited on ITO surface by cyclic voltammetry and chronoamperometry in acid medium. The film grown by cyclic voltammetry presented a flatter morphology and a detection limit of $0.05 \mathrm{~mol} \mathrm{~L}^{-1}$. The film grown by chronoamperometry presented interconnected network morphologywith a detection limit of 0.015 mol $\mathrm{L}^{-1}$ and a change of green color to blue more visually
\end{abstract}


perceptible. Thus, it is recommended to adopt chronoamperometry method to produce polyaniline films as colorimetric indicators for ammonia detection.

Keywords: Aniline. Polimerization; Indicator. Color change. Amnonia.

Recebido em: 30/01/2019 - Aprovado em: 30/03/2019 - Disponibilizado em: 15/07/2019

\section{INTRODUÇÂO}

Indicadores colorimétricos são compostos que permitem a obtenção de informações com manipulação mínima do sistema estudado. Os indicadores colorimétricos correlacionam os resultados de mudança de cor com outros parâmetros do ambiente ou do produto, tais como a temperatura e o frescor de alimentos. Os indicadores, diferentemente dos sensores não possuem um transdutor e a informação é dada diretamente através de mudança visual na cor. Estes indicadores podem ser inseridos as embalagens inteligentes na fase de produção ou fixados no interior ou no exterior desta após sua produção para o monitoramento da qualidade de alimentos.

O pescado é um produto de características intrínsecas bastante específicas que o caracterizam como alimento de alto teor nutritivo, porém de fácil deterioração (ARAÚJO et al., 2010). A concentração de aminas voláteis, conhecida como bases nitrogenadas voláteis é um dos parâmetros mais utilizados para avaliar a decomposição do pescado devido a sua simplicidade analítica e relação com o estado de frescor (CONTRERAS, 1994; ARAÚJO et al., 2010). Aminas voláteis, como trimetilamina, amônia e dimetilamina contribuem na quantidade conhecida como nitrogênio volátil total e são os compostos característicos responsáveis pelo odor de peixe e formadas após a fase inicial de frescura do peixe. Alguns sensores têm sido desenvolvidos explorando a mudança de $\mathrm{pH}$ decorrente da liberação de aminas voláteis (PACQUIT et al., 2007).

Sensores para voláteis são essencialmente de dois tipos, baseado em polímeros condutores orgânicos e metais inorgânicos (PANDEY, 2016). A polianilina (PANI) é um polímero condutor podendo ser facilmente dopado por protonação, isto é, sem que ocorra alteração no número de elétrons, durante sua oxidação ou na sua redução, associados à sua cadeia polimérica (MATTOSO, 1996). Huang et al. (1986) relacionaram o efeito eletrocrômico da PANI a seu estado de oxidação e protonação da sua macro molécula (Tabela 1). A propriedade de mudança de cor da PANI pode facilmente ser empregada para o desenvolvimento de indicadores colorimétricos de pescado. É possível a síntese de PANI por várias formas, por dispersões colidais, emulsões, polimerização interfacial, polimerização em solução e por meio da eletropolimerização, onde ela é facilmente sintetizada em meio ácido utilizando técnicas eletroquímicas. Em face da 
perceptível mudança de cor da PANI na presença de analito como a amônia, neste estudo avaliouse o uso de filmes de PANI depositados sobre superfície transparente de ITO $\left(\mathrm{In}_{2} \mathrm{O}_{3}: \mathrm{Sn}\right)$ para o desenvolvimento de um indicador colorimétrico para possível utilização em embalagens inteligentes na detecção da amônia.

Tabela 1- Descrição dos estados de oxidação da polianilina e suas características.

\begin{tabular}{l|l}
\hline Estado de oxidação & Estrutura \\
\hline Leucoesmeraldina & \\
\hline Sal de esmeraldina &
\end{tabular}

\section{METODOLOGIA}

\section{1- Produção do indicador colorimétrico}

Anilina foi previamente purificada por meio de destilação $\left(180^{\circ} \mathrm{C}, 5 \mathrm{~h}\right)$ e armazenada em frasco âmbar sob condições refrigeradas. Filme de polianilina foi eletropolimerizado no eletrodo de trabalho de vidro recoberto com ITO (Sigma Aldrich) com resistividade de 70-100 $\Omega$. Utilizou-se platina em espiral como eletrodo auxiliar e um eletrodo de $\mathrm{Ag} / \mathrm{AgCl}$ em $\mathrm{KCl} 3 \mathrm{~mol} \mathrm{~L}^{-1}$ como referência. Antes da eletropolimerização, o ITO foi previamente limpo com álcool etílico PA. A eletropolimerização ocorreu em meio de $\mathrm{H}_{2} \mathrm{SO}_{4} \quad 0,5$ mol L ${ }^{-1}$ e anilina destilada 0,2 mol L $\mathrm{L}^{-1}$ em uma célula eletroquímica de 10 $\mathrm{mL}$.

Foram escolhidas duas técnicas eletroquímicas para o desenvolvimento do filme, voltametria cíclica com faixa de potencial de $-0,2$ a $1,2 \mathrm{~V}$, a $10 \mathrm{mV} \mathrm{s}^{-1}$ por 2 ciclos de varredura e cronoamperometria a $0,75 \mathrm{~V}$ por 900 s.

\section{2- Detecção e quantificação da amônia}

Para o estudo de detecção da amônia foi montado um sistema com o auxílio de uma garrafa de vidro tampada, contendo um chumaço de algodão e com o indicador colorimétrico (filme de polianilina dopada - coloração verde) (Figura 1). Algodão foi umedecido no interior da garrafa com $1 \mathrm{~mL}$ de $\mathrm{NH}_{4} \mathrm{OH}$ em diferentes concentrações $(0,00015,0,0075,0,0085$, 0,015, 0,05 e $\left.15 \mathrm{~mol} \mathrm{~L}^{-1}\right)$. A mudança de cor do filme de polianilina foi avaliada com o auxílio de uma carta de cores (BIESALSKI et al., 1957) após 72h. O limite de detecção foi determinado a partir da menor concentração na qual houve mudança de cor do indicador. 


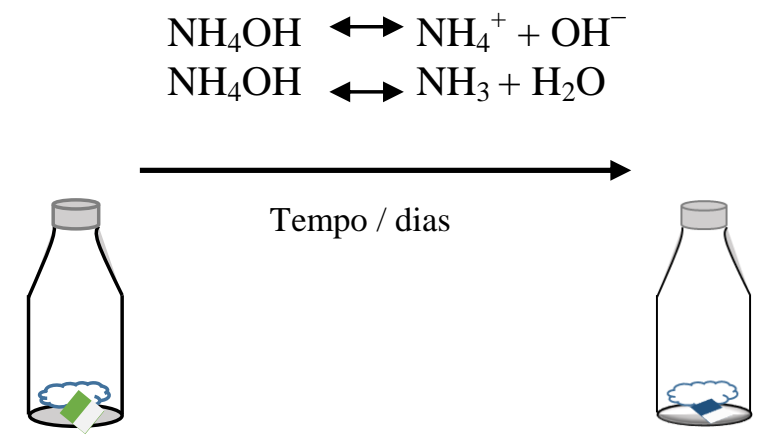

Figura 1: Esquema demonstrativo para a detecção da amônia.

\section{3- Análise morfológica do filme}

Filme foi montado sobre um stub com auxílio de uma fita adesiva condutora de carbono e observado em um microscópico eletrônico de varredura (Zeiss DSM 940 A). Foi utilizada a voltagem de aceleração de 20 KV para obtenção das imagens.

\section{3- RESULTADOS E DISCUSSÃO}

\section{1- Caracterização dos filmes de PANI}

As condições de eletropolimerização foram previamente otimizadas considerando a homogeneidade e adesão dos filmes a superfície de ITO.

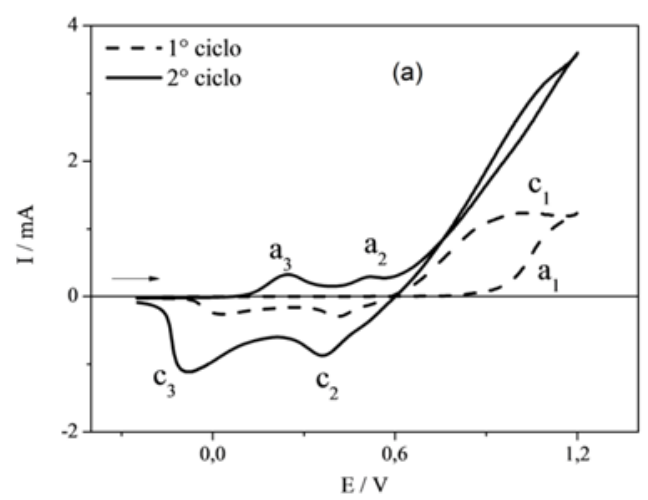

A condição de adesão foi estabelecida baseada na necessidade de que para uma aplicação comercial em alimentos, o filme polimerizado não deveria ser dessorvido da superfície com facilidade, pois poderia ter contato físico com a amostra e contaminá-la.

Em relação ao comportamento eletroquímico, o voltamograma do primeiro ciclo (Figura 2a) mostra o início do processo de oxidação da anilina em $0,8 \mathrm{~V}$ sobre superfície de ITO, com o aumento da corrente anódica, $\mathrm{a}_{1}$, correspondendo às reações de oxidação do monômero da anilina com conseguinte deposição do filme, $c_{1}$. Após o início da eletrodeposição é possível notar os pares redox $\mathrm{a}_{2}-\mathrm{c}_{2}$ e $\mathrm{a}_{3}-\mathrm{c}_{3}$, conforme descritos por Ferreira (2015) e Matos (2016).

A Figura $2 \mathrm{~b}$ apresenta o crescimento da polianilina a potencial constante, onde nos primeiros 150 segundos têm-se baixa corrente faradaica para a oxidação indicando a formação dos primeiros núcleos de polímeros na superfície. No decorrer do tempo há um recobrimento superficial com

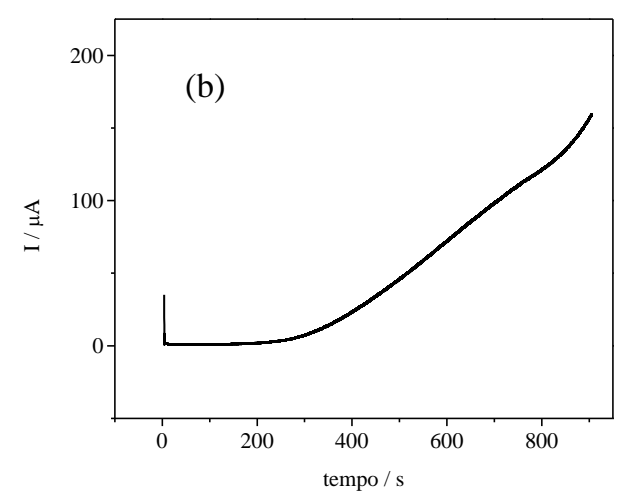

Figura 2: Crescimento de filme de polianilina sobre eletrodo de ITO em meio de $\mathrm{H}_{2} \mathrm{SO}_{4} 0,5$ mol L $\mathrm{L}^{-1}$ por voltametria cíclica a $10 \mathrm{mV} \mathrm{s}^{-1}$ (a e b) e por cronoamperometria a $0,75 \mathrm{~V}$ por $900 \mathrm{~s}$ (c e d). 
eletrodeposição de camadas sobre camadas aumentando a área exponencialmente, sendo a corrente elétrica proporcional à deposição de PANI-ES na superfície de ITO (COSTA, 2016).

O filme de PANI-ES crescido por voltametria cíclica apresentou uma superfície homogênea e mais plana (Figura 3a e 3b), no entanto, o filme crescido por cronoamperometria apresentou uma morfologia de rede interconectada com a presença de aglomerações (Figura 3c e 3d).
Sabe-se que as condições de eletropolimerização influenciam a morfologia dos filmes obtidos, interferindo na forma como as cadeias se arranjam sobre o substrato (Mattos, 2016). Em trabalho de Zhou et al. (2018) com filme crescido em superfície de ITO/PET por voltametria cíclica, os filmes apresentaram uma morfologia granular enquanto o filme crescido por cronoamperometria apresentou morfologia mais compacta.

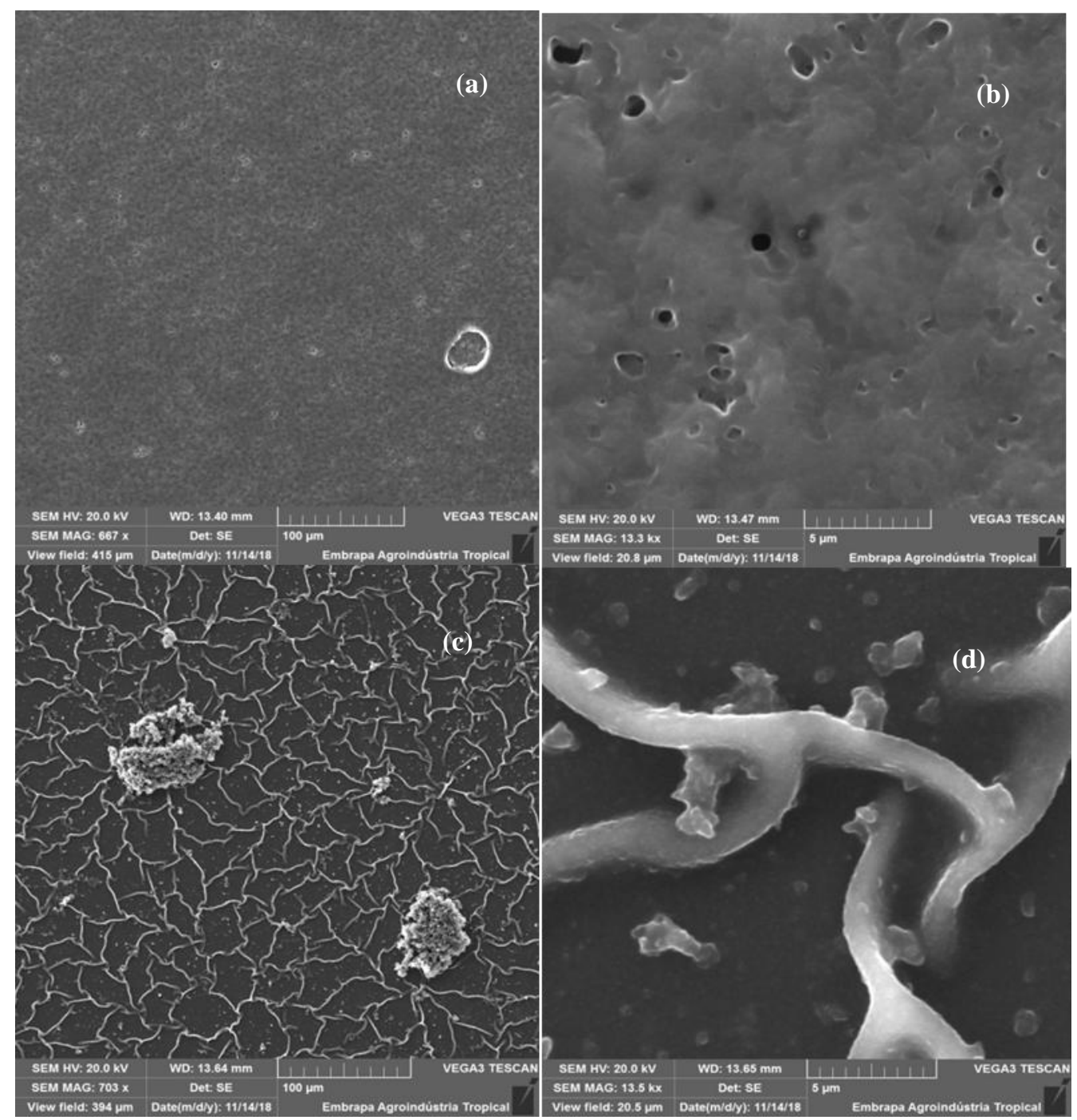

Figura 3. Imagens de MEV para filmes de polianilina crescidos por voltametria cíclica (A e B) ampliação de $703 x$ e 13,5 Kx, respectivamente, e por cronoamperometria (C e D) ampliação de 667x e 13,3 Kx, respectivamente. 


\section{2- Detecção da amônia}

A detecção foi realizada variando a concentração de $0,15 \mathrm{mmol} \mathrm{L}^{-1}$ a $15 \mathrm{~mol}$ $\mathrm{L}^{-1}$ de $\mathrm{NH}_{4} \mathrm{OH}$ a fim de determinar o limite de detecção do sensor de PANI-ES, crescidos por voltametria e por cronoamperometria, com base na mudança perceptível de cor. Observou-se a desdopagem da PANI-ES, que é verde, para a PANI-EB, que é azul e facilmente visível a olho nu. A Tabela 2 indica o limite de detecção para os diferentes filmes de polianilina.
O limite de detecção encontrado para o filme crescido por cronoamperometria foi de $0,015 \mathrm{M}$, muito próximo ao encontrado por Kuswandi et al. (2012), o qual foi de 0,01 M, utilizando filme de PANI crescido por via química. O limite de detecção do indicador colorimétrico encontrado neste trabalho foi adequado, principalmente, se compararmos com o desempenho de sensores elétricos bastante sensíveis baseado em mudança de resistividade, cuja sensibilidade foi de 50

Tabela 2: Determinação do limite de detecção da amônia para os filmes de PANI crescido por cronoamperometria e voltametria cíclica.

\begin{tabular}{|c|c|c|c|c|c|c|}
\hline $\begin{array}{l}{\left[\mathrm{NH}_{4} \mathrm{OH}\right]} \\
\text { Filmes }\end{array}$ & $\begin{array}{l}0,00015 \mathrm{~mol} \\
\mathrm{~L}^{-1}\end{array}$ & $\begin{array}{l}0,0075 \mathrm{~mol} \\
\mathrm{~L}^{-1}\end{array}$ & $\begin{array}{l}0,0085 \mathrm{~mol} \\
\mathrm{~L}^{-1}\end{array}$ & $\begin{array}{l}0,015 \mathrm{~mol} \\
\mathrm{~L}^{-1}\end{array}$ & $\begin{array}{l}0,05 \quad \mathrm{~mol} \\
\mathrm{~L}^{-1}\end{array}$ & $\begin{array}{ll}15 & \mathrm{~mol} \\
\mathrm{~L}^{-1} & \end{array}$ \\
\hline $\begin{array}{l}\text { Filmes } \\
\text { eletropolimerizados } \\
\text { por Voltametria } \\
\text { cíclica }\end{array}$ & $x$ & $x$ & $x$ & $x$ & $\checkmark$ & $\checkmark$ \\
\hline $\begin{array}{l}\text { Filmes } \\
\text { eletropolimerizados } \\
\text { por } \\
\text { cronoamperometria } \\
900 \text { s }\end{array}$ & $x$ & $x$ & $x$ & $\checkmark$ & $\checkmark$ & $\checkmark$ \\
\hline
\end{tabular}

A mudança de cor foi monitorada com o auxílio de uma carta de cores. A carta de cores de Biesalski et al. (1957) é uma ferramenta para identificação de cores onde é reunida uma série de padrões de cor. No canto superior de cada padrão de cor há uma abertura onde são posicionadas as amostras para se fazer a comparação visual (Figura 4). É possível notar que a mudança de cor do filme crescido por cronoamperometria é mais perceptível que aquele crescido por voltametria cíclica. ppm, enquanto um sensor comercial apresentou uma sensibilidade de 150 ppm para a detecção de amônia (MATINDOUST et al., 2017).

$\mathrm{Na}$ literatura existem trabalhos que utilizaram a PANI como indicador para o monitoramento da deterioração da tilápia. Domínguez-Aragón et al. (2018) desenvolveram o indicador, copolímero poli (orto-fenilenodiamina-co-anilina), pelo método de "spray coating", tendo sintetizado a PANI por método químico. No referido 

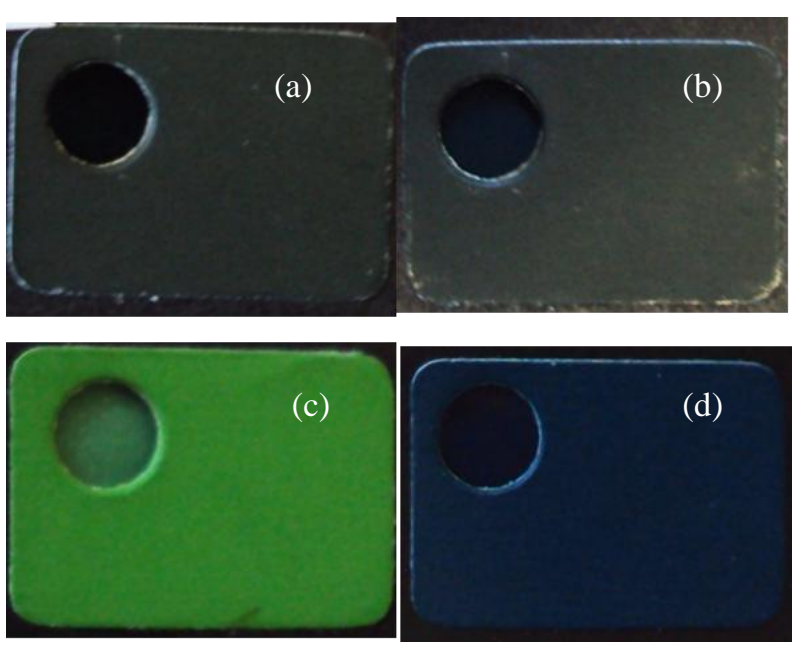

Figura 4: Imagens das cores dos filmes de PANI-ES crescidos por voltametria cíclica (4a) e cronoamperometria (4c); filmes de PANI-EB crescidos por voltametria cíclica (4b) e cronoamperometria (4d) de acordo com o uso de uma carta de cores. Códigos na carta de cores de U 22:2:7, L 23:7:3, U 19:1:7, W 19:4:6, respectivamente para os filmes.

trabalho a cor do indicador variou de vermelho para amarelo, onde o $\mathrm{pH}$ teve um papel importante. Em trabalho de Wang et al. (2018), o indicador colorimétrico foi obtido através de síntese química, e a cor do indicador variou de verde para azul, sendo aplicado diretamente no peixe para a detecção das amônias voláteis. Vale ressaltar que este tipo de metodologia não é a mais adequada, pois o contato do indicador com o alimento representa um risco de contaminação partindo do pressuposto que a anilina é um composto com características tóxicas e deve-se manter o isolamento do alimento.

Apesar de existir trabalho com indicador colorimétrico com PANI para ava- liar a deterioração de pescados, os trabalhos relatados sintetizaram PANI por meio químico, onde não há reprodutibilidade do filme, é gasto um tempo maior de síntese e gerado uma maior quantidade de resíduos químicos e nenhum dos trabalhos apresentou um limite de detecção dos indicadores. Neste trabalho filme de PANI sintetizado por via eletroquímica teve a vantagem de diminuir o tempo para o desenvolvimento do filme, não necessitou de catalisadores e gerou menos resíduos químicos.

\section{4- CONCLUSÃO}

Foi possível produzir um indicador colorimétrico a partir da polianilina por eletropolimerização, uma técnica que produz filmes reprodutíveis e gera menos resíduos químicos. O filme crescido por voltametria cíclica apresentou uma morfologia mais uniforme, porém apresentou um limite de detecção maior que o filme crescido por cronoamperometria, além de ser menos perceptível visualmente a mudança de cor referente à mudança no estado de oxidação de PANI-ES para PANI-EB. Assim recomenda-se eletropolimerizar o filme por cronoamperometria para a produção de um indicador colorimétrico para detecção da amônia.

\section{REFERÊNCIAS}

ARAÚJO, D. A. F. V., SOARES, K. M. P. e GÓIS, V. A. Características gerais, processos 
de deterioração e conservação do pescado.

PUBVET, v. 4, n. 9, art.771, 2010.

BIESALSKI, E.; MAATSCH, R.; BENARY, F. E. Pflanzenfarben-Atlas fur Gartenban, Landwirtschaft und Fortswesen mit Farbzeichen nach Din 6164.

Gottingen,Musterschmidt-Verlag, 1957. 31p.

CONTRERAS-GUZMÁN, E. S. Bioquímica de pescados e derivados. Jaboticabal: FUNEP, 1994. 409 p.

COSTA, J. C. M. Síntese e caracterização galvânica da Polianilina (PANI) para aplicação em supercapacitores. 2016. 75f. Dissertação (Mestrado em ciências e engenharia de materiais)- Universidade Federal do Amazonas, Amazonas.

DOMÍNGUEZ-ARAGÓN, A.; OLMEDOMARTÍNEZ, J. A.; ZARAGOZACONTRERAS, E. A. Colorimetric sensor based on a poly (ortho-phenylenediamine-coaniline) copolymer for the monitoring of tilapia (Orechromis niloticus)

freshness.Sensors and Actuators B:

Chemical, v. 259, p. 170-176, 2018.

\section{FERREIRA, L. L. Estudo}

espectroeletroquímico de politiofenos substituídos para aplicação em dispositivos eletrocrômicos. 2015. $76 \mathrm{f}$. Dissertação (Mestrado em Química)Universidade Federal de Minas Gerais, Minas Gerais.

HUANG, W.; HUMPHREY, B. D.; MACDIARMID, A. G. Polyaniline, a novel conducting polymer. Morphology and chemistry of its oxidation and reduction in aqueous electrolytes.Journal of the Chemical Society, Faraday Transactions 1: Physical Chemistry in Condensed Phases, v. 82, n. 8, p. 2385-2400, 1986.

KUSWANDI, A, B.; RESTYAN, J. A.; ABDULLAH, A.; HENG, L.Y.; AHMAD, M. A novel colorimetric food package label for fish spoilage based on polyaniline film Food Control. V. 25, p. 184-189, 2012.
MATINDOUST, S.; FARZI, A.; NEJAD, M.B.; HADI, M.; ABADI, S.; ZOU, Z.; ZHENG, R. Ammonia gas sensor based on flexible polyaniline films for rapid detection of spoilage in protein-rich foods. Journal of Materials Science: Materials in

Electronics. Disponível em $<$ https://link.springer.com/article/10.1007/s1 0854-017-6471-z > . Acesso em 30 de janeiro de 2019.

\section{MATTOS, L. L. Eletrodos de polianilina} para aplicação em pseudocapacitores. 2016. 148f. Tese (doutorado em Física) Universidade Federal de Santa Catarina, Sanata Catarina.

MATTOSO, L. H. C. Polianilinas: síntese, estrutura e propriedades. Química nova, v. 19, n. 4, p. 388-399, 1996.

PACQUIT, A.; LAU, K.T.; MCLAUGHLIN, H.; FRISBY, J.; QUILTY B.; DIAMOND, $D$. Development of a volatile amine sensor for the monitoring of fish spoilage. Talanta, v. 69, p. 515-520, 2006.

PANDEY, S. Highly sensitive and selective chemiresistor gas/vapor sensors based on polyaniline nanocomposite: A comprehensive review. Journal of Science: Advanced Materials and Devices, v. 1, p.431-453, 2016.

WANG, W.; LI, M.; LI, H.; LIU, X.; GUO, T.; ZHANG, G.; XIONG, Y. A renewable intelligent colorimetric indicator based on polyaniline for detecting freshness of tilapia. Packaging Technology and Science, v. 31, n. 3, p. 133-140, 2018.

ZHOU, K.; WANG, H.; JIU, J.; LIU, J.; SUGANUMA, K. Polyaniline films with modified nanostructure for bifunctional flexible multicolor electrochromic and supercapacitor applications. Chemical Engineering Journal, v. 345, p. 290-299, 2018. 


\section{Raíssa Costa de Oliveira}

Bacharel em Química pela Universidade Federal do Ceará, Fortaleza- CE.

\section{Francisco Belmino Romero}

M. Sc. Em Ciência dos Materiais e Engenharia Metalúrgica, professor no Departamento de Química da Universidade Federal do Ceará, Fortaleza- CE.

\section{Carlucio Roberto Alves}

Dr. em físico-química, professor no Departamento de Química da Universidade Estadual do Ceará, Fortaleza- CE.

\section{Atanu Biswas}

Pesquisador do Departamento de Agricultura dos Estados Unidos, Peoria- Illinois.

\section{Huai N.Cheng}

Pesquisador do Departamento de Agricultura dos Estados Unidos, Nova Orleans- Luisiana

\section{Roselayne Ferro Furtado}

Dra. em Biotecnologia, pesquisadora da Embrapa Agroindústria Tropical, Fortaleza- CE. 